\title{
Combining selection valve and mixing chamber for nanoflow gradient generation: toward developing a liquid chromatography cartridge coupled with mass spectrometer for protein and peptide analysis
}

\author{
Apeng Chen ${ }^{1}$ \\ ${ }^{1}$ Department of Chemistry and Biochemistry, University of Oklahoma \\ Norman, OK 73019, USA \\ Tel.: +1 (405) 325 5645, Fax: +1 (405) 325 6111, E-mail: apeng.chen@,ou.edu \\ Joann J. $\mathrm{Lu}^{1}$ \\ ${ }^{1}$ Department of Chemistry and Biochemistry, University of Oklahoma \\ Norman, OK 73019, USA \\ Tel.: +1 (405) 325 5645, Fax: +1 (405) 325 6111, E-mail: joannlu_liu@yahoo.com \\ Congying $\mathrm{Gu}^{1,2}$ \\ ${ }^{1}$ Department of Chemistry and Biochemistry, University of Oklahoma \\ Norman, OK 73019, USA \\ Tel.: +1 (405) 325 5645, Fax: +1 (405) 325 6111, E-mail: congyinggu@ hotmail.com \\ ${ }^{2}$ Veritas Laboratories, LLC, Tifton, GA 31793, USA \\ Min Zhang ${ }^{1}$ \\ ${ }^{1}$ Department of Chemistry and Biochemistry, University of Oklahoma \\ Norman, OK 73019, USA \\ Tel.: +1 (405) 325 5645, Fax: +1 (405) 325 6111, E-mail: Min.Zhang@utas.edu.au \\ Kyle B. Lynch ${ }^{1}$ \\ ${ }^{1}$ Department of Chemistry and Biochemistry, University of Oklahoma \\ Norman, OK 73019, USA \\ Tel.: +1 (405) 325 5645, Fax: +1 (405) 325 6111, E-mail: 1ynchkb@,ou.edu \\ Shaorong $\mathrm{Liu}^{1} *$ \\ ${ }^{1}$ Department of Chemistry and Biochemistry, University of Oklahoma \\ Norman, OK 73019, USA \\ Tel.: +1 (405) 325 9013, Fax: +1 (405) 325 6111, E-mail: $\underline{\text { Shaorong.liu@,ou.edu }}$
}

*Corresponding author 


\begin{abstract}
Toward developing a micro HPLC cartridge, we have recently built a high-pressure electroosmotic pump (EOP). However, we do not recommend people to use this pump to deliver an organic solvent directly, because it often makes the pump rate unstable. We have experimented several approaches to address this issue, but none of them are satisfactory. Here, we develop an innovative approach to address this issue. We first create an abruption (a dead-volume) within a fluid conduit. We then utilize an EOP to withdraw, via a selection valve, a train of eluent solutions having decreasing eluting power into the fluid conduit. When these solutions are further aspirated through the deadvolume, these solutions are partially mixed, smoothening concentration transitions between two adjacent eluent solutions. As these solutions are pushed back, through the dead-volume again, a smooth gradient profile is formed. In this work, we characterize this scheme for gradient formation, and we incorporate this approach with a highpressure EOP, a nanoliter injection valve, and a capillary column, yielding a micro HPLC system. We then couple this micro HPLC with an electrospray ionization - mass spectrometer for peptide and protein separations and identifications.
\end{abstract}

Keywords: Electroosmotic pump, nanoflow, gradient generator, high performance liquid chromatography, mass spectrometry 


\section{Introduction}

Electroosmotic pump (EOP) [1-4] is one of the most promising micropumps that can be used for HPLC due to its compact size, and low fabrication cost. We have recently developed a high-pressure EOP [5-8] and incorporated it into a miniaturized HPLC system for protein and peptide separations $[6,7,9,10]$. Implementing a gradient elution using this EOP has been a challenge, because its pump rate becomes unstable when we use it to pump a high-organic-content solvent $[9,10]$. We have experimented three approaches for alleviating this issue but none of them were completely satisfactory $[7,9$, 10]. In one approach [7], we preloaded two eluent segments consecutively into a capillary (with the weaker eluent in front), and this capillary was then connected to the outlet of the EOP. As the EOP drove the eluent segments forward, the two solutions were mixed, forming a gradient eluent at the interfacial region. We then used this gradient to perform peptide separations. Apparently, precisely tuning the gradient profile using this method is difficult.

In the second approach [9], we utilized a ten-port switching valve connected with two injection loops for gradient delivery. A similar scheme had been described earlier by others [11]. In one position, while a weaker eluent in loop 1 was delivered for analyte elution, a stronger eluent was loaded into loop 2. As the valve was switched to the other position, the eluent in loop 2 was delivered for elution, while loop 1 was loaded with the next stronger eluent. Since these operations could be repeated continuously, a desired gradient could be produced. The downside of this approach is that the gradient created 
has a step-profile. Its operations are tedious, especially when a close-to-linear gradient is desired.

In the third approach [10], we constructed a binary EOP for gradient production. The working principle of a binary EOP gradient generator (bi-Egg) was identical to that of a conventional binary gradient pump. Owing to the nature of electroosmotic pumping, a backflow was created whenever there was a head pressure. This backflow complicated the bi-Egg's operation. To address this issue, we developed formulas to tune the voltages on both EOPs to achieve desired gradient. However, due to the viscosity change with eluent composition, the pressure drop across the column (or the head pressure of the pump) varied, leading to deviations between actual and programmed gradient profiles.

In this work, we develop a novel approach to address this issue. Fig. 1 presents the basic idea of this approach. We first create an abruption (a dead-volume) within a fluid conduit (see Fig. 1A) by inserting a piece of larger inner diameter (i.d.) of polyetheretherketone (PEEK) tubing between two pieces of smaller i.d. capillaries (see Fig. 1B). We then aspirate a series of eluent solutions having different elution strengths, via a selection valve, into this capillary assembly, and through the PEEK tubing. At a predetermined position (or time), we push these solutions back, through the PEEK tubing again, to a targeted location (e.g., an HPLC column). During the course of these solution movements (back-and-forth movement inside the capillary assembly and twice through the dead-volume), smooth concentration interfaces are formed between adjacent eluent solutions, creating a smooth gradient profile. Because the number of eluent solutions, the concentrations and volumes of the eluent solutions, the size of the dead-volume, and the pattern (velocity, duration, or movement style) of the solution movement can be 
conveniently tuned and controlled, any desired gradient profile can be produced. In this experiment, we characterize this approach for smooth gradient generation. We incorporate such a gradient generator with a high-pressure EOP, a nanoliter injection valve, and a capillary column into a compact HPLC platform. We further couple this HPLC with an electro-spray-ionization (ESI) - mass spectrometer (MS) for peptide and protein separation and identification.

\section{Materials and methods}

\subsection{Reagents and Materials.}

Ethylene glycol dimethacrylate (EDMA, 98\% w/w, hereinafter " $\%$ " indicates “\% w/w" unless otherwise stated) and butyl methacrylate (BMA) were purchased from Alfa Aesar (Ward Hill, MA). 1-propanol and 2,2'-azobisiso-butyronitrile (AIBN, 98\%) was bought from Aldrich (Steinheim, Germany). Methacryloyloxypropyl-trimethoxysilane ( $\gamma$ MAPS, 98\%) was obtained from Acros (Fairlawn, NJ). Acrylamide, N,N'-Methylene bisacrylamide (bis), N,N,N',N'-Tetramethylethylenediamine (TEMED) and ammonium persulfate (APS) were purchased from Bio-Rad Laboratories (Hercules, CA). 1,4Butanediol (99\%) was purchased from Emerald BioSystems (Bainbridge Island, WA). Proteomics grade modified trypsin was obtained from Promega (Madison, WI). LC-MS grade acetonitrile (ACN) was bought from Fisher Scientific (Fair Lawn, NJ). 2Acrylamido-2-methylpropane sulfonic acid (AMPS, 99\%), bovine serum albumin (BSA), sodium hydroxide, ammonium bicarbonate, and sodium acetate were purchased from Sigma-Aldrich (St. Louis, MO). Fused silica capillaries were obtained from Polymicro 
Technologies (Phoenix, AZ). Hydrochloric acid was obtained from EMD Millipore

(Darmstadt, Germany). All solutions were prepared with ultra-pure water purified by a NANO pure infinity ultrapure water system (Barnstead, Newton, WA).

\subsection{Preparation of EOP monolithic columns.}

The preparation protocol was similar to what we had described previously [9] but with some minor modifications. A capillary of $100 \sim 150 \mathrm{~cm}(100 \mu \mathrm{m}$ i.d. $\times 360 \mu \mathrm{m}$ o.d. $)$ was first vinlyized using the following procedure. Briefly, the capillary was first flushed with acetone and $1.0 \mathrm{M} \mathrm{NaOH}$, each for $10 \mathrm{~min}$. After the capillary was filled with $1.0 \mathrm{M}$ $\mathrm{NaOH}$ and its ends were sealed by rubber septa, it was placed in an oven at $100{ }^{\circ} \mathrm{C}$ for 2 h. The capillary was then taken out from the oven, and it was flushed with water, $0.1 \mathrm{M}$ $\mathrm{HCl}$, and acetone; each for $20 \mathrm{~min}$. After the capillary was dried with $\mathrm{N}_{2}$ at $60 \mathrm{psi}$ for $1 \mathrm{~h}$, the capillary was filled with $30 \%(\mathrm{v} / \mathrm{v}) \gamma$-MAPS in acetone, and its ends were sealed with septa, and then the capillary was put back in the oven at $50{ }^{\circ} \mathrm{C}$ for $14 \mathrm{~h}$. The capillary was vinylized and ready for the monolith preparation after being rinsed with acetone and dried with $\mathrm{N}_{2}$ at 60 psi for $2 \mathrm{~h}$.

The vinylized capillary was then cut to $16-\mathrm{cm}$-long segments. After degassing by purging with helium for $\sim 5 \mathrm{~min}$, a solution containing $10.0 \mathrm{mg}$ AMPS, $230.0 \mathrm{mg}$ BMA, $160.0 \mathrm{mg}$ EDMA, $4.0 \mathrm{mg}$ AIBN, $424.0 \mathrm{mg}$ 1-propanol, $116.0 \mathrm{mg}$ 1,4-butanediol and 60.0 uL water was loaded into a capillary segment. A 500 600 psi pressure supplied by a nitrogen cylinder was applied to the ends of the capillary, and the capillary was placed in a water bath at $60{ }^{\circ} \mathrm{C}$ for polymerization for $20 \mathrm{~h}$. After approximately $2 \mathrm{~cm}$ of the capillary ends was trimmed, the monolithic column was flushed first with acetonitrile at 
1500 2000 psi for overnight using an HPLC pump to remove unreacted monomers and other chemicals, and then with a $3.0 \mathrm{mM}$ sodium acetate solution ( $\mathrm{pH} 5.0)$.

\subsection{Preparation of monolithic polystyrene-divinylbenzene (PS-DVB) columns.}

The preparation was based on a protocol developed by Premstaller et al. [12]. A 90.0cm-long vinlylized fused silica capillary (360 $\mu$ m o.d., $150 \mu$ i.d.) was filled with a helium-purged solution containing $45.31 \mathrm{mg}$ styrene, $45.70 \mathrm{mg}$ divinylbenzene, 107.77 $\mathrm{mg}$ decanol, $17.78 \mathrm{mg}$ tetrahydrofuran (THF), and $2.50 \mathrm{mg}$ of AIBN. After both ends of the capillary were blocked, the capillary was placed into a water bath at $60^{\circ} \mathrm{C}$ for 24 hours to allow the monolith formation. The capillary was then cut into two halves (two columns), and the columns were washed with acetonitrile (ACN) for $\sim 5 \mathrm{~h}$ at $\sim 1000 \mathrm{psi}$ to remove the unreacted monomers.

\subsection{Apparatus.}

Fig. 2A presents a schematic configuration of the HPLC system. It consists of two EOPs, a 12-port switching valve (V1, Valco Instruments, Houston, TX), a 7-port stream selector (V2, Valco Instruments), a 60-nL or a 10-nL injection valve (V3, Valco Instruments), a packed capillary column ( $100 \AA, 3 \mu \mathrm{m}, 75 \mu \mathrm{m} \times 100 \mathrm{~mm}$, Waters, Atlantis dc18 NanoEase Column) or a home-made PS-DVB monolith column (C), and a Linear UVIS 200 absorbance detector (D, Linear Instruments, Reno, NV). Each EOP was composed of three parallel monolithic columns; these columns were connected in parallel via two micro-crosses (Valco Instruments). The high voltage was applied to the monolithic columns via two micro-tees (Valco Instruments), each being attached with a bubbleless electrode. The protocols for preparing the bubbleless electrode [5] [see also 
Supporting Information (SI) for details] and assembling the EOP [10] were reported previously. The Linear UVIS 200 absorbance detector was set at $214 \mathrm{~nm}$ for peptide and protein separations and $265 \mathrm{~nm}$ for gradient profile measurements. An NI multifunctional card DAQ Card (6062E, National Instruments, Austin, TX) was used to acquire the absorbance signal, and the signal was processed by an in-house written LabView program.

\subsection{Preparation of peptide and protein samples.}

Myoglobin samples were obtained from Drs. Yi and Richter-Addo's lab [13]. Briefly, gene engineered horse heart mutant myoglobin (hh Mb, H64V) was expressed in E.coli, followed by ion-exchange chromatography purification. Other proteins were purchase from Sigma-Aldrich and used without further purifications.

To prepare a protein stock solution for trypsin digestion, a protein was dissolved in $100 \mathrm{mM}$ ammonium bicarbonate to a concentration of $50 \mathrm{mg} \mathrm{mL}^{-1}$. To prepare for digestion, appropriate volumes of water and $100 \mathrm{mM}$ ammonium bicarbonate were added to dilute a protein stock solution to a final concentration of $10 \mathrm{mg} \mathrm{mL}^{-1}$ protein in $50 \mathrm{mM}$ ammomium bicarbonate. After $1 \mu \mathrm{L}$ of $10 \mathrm{mM}$ DTT (freshly-made) was added, trypsin was introduced to the solution at a substrate-to-enzyme ratio of 100:1 to digest the proteins at $37^{\circ} \mathrm{C}$ for $8 \mathrm{~h}$. One microliter of $1.0 \mathrm{M} \mathrm{HCl}$ was added to the solution to terminate the digestion. After the tryptic digests were processed by passing it through an ULTRAFREE $^{\circledR}$-MC 5,000 NMWL Filter Unit (Merck-Millipore, Germany), it was ready for separation, or it was stored at $-20{ }^{\circ} \mathrm{C}$.

\subsection{HPLC separation.}


Referring to Fig. 2A, one of the six ports of V2 was connected to waste (W), and the other five were connected eluents 1 through 5 (E1 through E5). The compositions of E1, E2, E3, E4, and E5 were respectively $45 \%, 27.5 \%, 15 \%, 10 \%$, and $5 \% \mathrm{ACN}$ in $0.1 \%$ TFA. When the top EOP was connected to the column (C) as shown in Fig. $2 \mathrm{~A},+3.2 \mathrm{kV}$ was applied to the pump (the pump rate at this high voltage was $\sim 150 \mathrm{~nL} \mathrm{~min}{ }^{-1}$ ). The EOP drove mobile phase A (MP A, composition 5\% ACN in 0.1\% TFA) to equilibrate $\mathrm{C}$ for sample injection. At the same time, $+3.6 \mathrm{kV}$ was applied to the bottom EOP for a 10 min to rinse the gradient loop to waste (W). The pump rate under this condition was $\sim 500$ $\mathrm{nL} \min ^{-1}$. Higher voltage could be applied to increase flow rate; however, we did not use voltages higher than $10 \mathrm{kV}$ to avoid excessive Joule heating. Then, $-3.6 \mathrm{kV}$ was applied to the pump, and V2 was switched to E1, E2, E3, E4, and E5 for $12 \mathrm{~min}, 9.75 \mathrm{~min}, 7.8$ $\mathrm{min}, 3.75 \mathrm{~min}$, and $5.5 \mathrm{~min}$, respectively. The pump rate at $-3.6 \mathrm{kV}$ was about $\sim-500 \mathrm{~nL}$ $\min ^{-1}$. Then, $-3.6 \mathrm{kV}$ was turned off, and five eluent solutions were parked inside the gradient loop. A $10-\mathrm{nL}$ injection valve was used to inject $10 \mathrm{~nL}$ of a sample, or a $60-\mathrm{nL}$ injection valve and a time-controlled injection scheme ( 8 seconds at a flow rate of $\sim 150$ $\mathrm{nL} \min ^{-1}$ ) was used to inject $\sim 20 \mathrm{~nL}$ of a sample into the column for separation. After sample injection, V1 was switched to the other position. The top EOP drove the gradient eluent through V3 and $\mathrm{C}$ for chromatographic elution. At this time, $-3.6 \mathrm{kV}$ was applied to the bottom EOP to aspirate MA for 15 20 min, and the high voltage was turned off. After the separation was complete, V1 was switched back to initiate a new run.

\section{Results and discussion}




\subsection{Nano-flow gradient generation.}

To test whether an EOP element can form smooth gradient profile easily, we setup a gradient generator as schematically shown in Fig. 1A. The PEEK tubing had an i.d. of 390- $\mu$ m-i.d., and it was inserted in between two pieces of $75-\mu$ m-i.d. capillaries; one had a length of $5 \mathrm{~cm}$, and the other had a length of $15 \mathrm{~cm}$ (see Fig. 1B). The free end of the 5-cm-capillary was then connected to an EOP, while the free end of the $15-\mathrm{cm}-$ capillary was connected to the common port of a selection valve. Five solutions having different acetonitrile concentrations were connected to five ports of the selection valve, and a waste reservoir was connected to the last port of the selection valve. A Linear UVIS 200 absorbance detector was set at $265 \mathrm{~nm}$ and affixed to the 15 -cm-capillary to monitor the gradient as the solution passed across the detector.

When equal lengths of solutions were aspirated in and pushed out through the capillary assembly, a smoother concentration transition was observed for the last two exiting solutions (normal concentrations $\sim 75 \%$ and $\sim 100 \%$ ), compared to the earlier two exiting solutions (normal concentrations $\sim 25 \%$ to $\sim 50 \%$ ). The reason was that the last two exiting solutions were the first two solutions aspirated in and they had travelled a longer distance and mixed more completely than the earlier exiting solutions. There are two ways to compensate the different level of mixing; to aspirate a longer solution segment for an earlier aspirated solution, and/or to reduce the concentration difference between the later aspirated solutions. Fig. $1 \mathrm{C}$ presents the results by reducing the solution segments for the later aspirated solutions; a good linear gradient was created. Increasing the dead-volume can also improve the smoothness of the gradient profile (see Fig. S1). It 
is worth pointing out that the mixing chamber volume for a 10 -mm-long and $390-\mu \mathrm{m}-\mathrm{i} . \mathrm{d}$. capillary was $\sim 1.2 \mu \mathrm{L}$.

With the above knowledge, we set up an apparatus as presented in Fig. 2A. A deadvolume was created between V1 and V2 (the thick line M on the connection capillary) or within the Gradient Loop, and an UV absorbance detector was affixed between V1 and V3 to monitor the gradient profile. The smoothness of the gradient profile did improve but not significantly. The reason was that a dead volume of $2 \sim 3 \mu \mathrm{L}$ was present in $\mathrm{V} 1$ as the eluents passed through 2 ports from V2 to the Gradient Loop and 2 ports from the Gradient Loop to V3; this dead volume served effectively as a mixing chamber. Therefore, for the rest of this experiment, we did not include any additional dead-volume in the apparatus. However, it is preferable to put a mixing chamber after EOP when a relatively large total volume of eluents (e.g., more than $20 \mu \mathrm{L}$ ) and/or a shadow gradient profile is needed for a long-time separation, such as for proteomics analysis, which often requires the resolution of more than one thousand peaks, since the inherent dead-volume of the valve is not enough to generate a smooth gradient profile, especially when a "zero dead-volume" valve that have been recently developed by Valco Instruments is used.

The very first solution aspirated was usually the strongest eluent, and we often aspirated a large volume of this solution so that we could wash the column thoroughly after the separation. We used another solution with the lowest elution power called mobile phase A (or MA) stored in the other Loop to equilibrate the column for the next separation. 
The volumes of the Gradient loop and the MA loop should be adequate so that no eluent solutions or MA solution could be aspirated into the EOPs.

\subsection{Performance of nano-flow gradient generator.}

To test whether we could produce reproducible gradients, we utilized the apparatus as shown in Fig. 2A, but without V3 and C, to produce gradient profiles repetitively. Fig. 2B and Fig.S2 present the results, which show that reproducible gradient profiles were generated. The average standard deviations of the acetonitrile concentration profiles were less than $1 \%$.

We then incorporated the nanoliter injection valve (V3) and the separation column (C) into a micro HPLC platform. We used this platform for repetitive separations of peptides (trypsin-digested bovine serum albumin); very reproducible chromatograms were produced (see Fig. 3).

To examine the validity of the above system, we compared its performances with the performances of a commercially available Agilent 1200 HPLC system (Agilent Technologies, Santa Clara, CA). We first used the micro HPLC platform to separate tryptic digests of bovine serum albumin and hh Mb H64V. The same samples were then separated on the Agilent system. These results were presented in Fig. 4A-4D. By visualizing the chromatograms, we could say that comparable efficiencies and resolutions were obtained. The two sets of results did exhibit some differences, however, and these differences could be attributed partially to the gradient profile differences. 
It is also important to notice that there was a $\sim 12$-min time delay from the programmed gradient profile to the actual gradient profile using the Agilent 1200 HPLC system. Using our micro HPLC system, this time delay was shortened to $3 \sim 5 \mathrm{~min}$.

\subsection{Coupling micro HPLC platform with mass spectrometer for protein separation and identification.}

Proteins play the most important physiological functions in cells and tissues, and any mutation or mutations in a protein may cause a disease, since a mutated protein can lead to its incorrect three-dimensional folding and subsequently cause malfunction of the protein [14]. The development of mass spectrometry allows us to distinguish a single protein from its isoforms and other proteins, lending us an effective tool for characterizing human diseases and ultimately for developing therapies to treat these diseases [15]. Myoglobin is well-known as an oxygen carrier. It also removes nitrite $\left(\mathrm{NO}_{2}{ }^{-}\right)$by reducing it to nitric oxide (NO) and lowers the energy status of heart cells, which subsequently prevents heat injury from hypoxic conditions $[16,17]$. It was reported that a single amino acid mutant of horse heart myoglobin (hh Mb, H64V) dramatically decreased the efficiency of $\mathrm{hh} \mathrm{Mb}$ as a scavenger to remove $\mathrm{NO}_{2}^{-}$, due to the mutation that changed the H-bonding in the distal pocket of the myoglobin [13]. Here, we coupled our nano-HPLC with a mass spectrometer (LCQ DECA XP plus, Thermo Fisher Scientific Inc., Waltham, MA) for protein separation and identification. Detailed interfacing between micro HPLC and ESI-MS was presented in Fig. S3. 
Fig. 5 presents a chromatogram for a mixture of wild type horse heart myoglobin (wt hh $\mathrm{Mb}$ ), a mutated hh Mb H64V, and the hh Mb H64V missing the first methionine [18]. Peak a is the wt hh Mb. The mass spectrometer (MS) measured molecular weight is $16,951.76 \mathrm{Da}$ (see inset A), which is consistent with its theoretical value (16951.49 Da [19]). Peak $\mathrm{c}$ is the mutated hh $\mathrm{Mb} \mathrm{H} 64 \mathrm{~V}$, which has one additional methionine at the $\mathrm{N}$ end and its histidine at position 64 is mutated to valine. The MS measured molecular weight is $17,044.81 \mathrm{Da}$ (see inset C), which is consistent with the calculated value (17044.67 Da). Peak b is the mutated hh Mb H64V missing the first methionine [18]. The MS measured molecular weight is $16,913.50 \mathrm{Da}$ (see inset $\mathrm{B}$ ), which is consistent with calculated value (16913.48 Da). In other words, all proteins could be positively identified with this approach.

\section{Conclusions}

We have developed an innovative approach for smooth gradient generation. The approach combines an EOP with a selection valve to withdraw a series of solutions having decreasing eluting power into a fluid conduit that comprises of a mixing chamber (in this work the inherent dead-volumes of V1 and V2 were used as the mixing chamber). We have incorporated this approach with a high-pressure EOP, a nanoliter injection valve, and a packed capillary column into a micro HPLC system. Theoretically, any type of desired gradient profile can be generated since it is determined by pre-defined eluents. To make the process automated, we are developing an auto-pump controlled by an inhouse written system, and the result would be published somewhere else. We have further coupled the micro HPLC with an ESI-MS for peptide and protein analysis. 
Portability is potentially a highly desirable feature for remote field and point-of-care applications [20,21]. At the time being, the repeatability of our micro HPLC is not as good as the Agilent 1200 system, but it will improve as a more rigid apparatus is developed. Because the micro HPLC could be produced at a cost of $\$ 3-4 \mathrm{k}$, compared to a commercial HPLC system that costs $\$ 30-40 \mathrm{k}$, the micro HPLC holds great promises in these applications.

\section{Acknowledgements}

This work is partially sponsored by DOE through DE-SC0006351 and the Oklahoma Center for the Advancement of Science and Technology through AR11-003.

\section{References}

[1] L.X. Chen, J.P. Ma, Y.F. Guan, An electroosmotic pump for packed capillary liquid chromatography, Microchem. J., 75 (2003) 15-21.

[2] L.X. Chen, J.P. Ma, Y.F. Guan, Study of an electroosmotic pump for liquid delivery and its application in capillary column liquid chromatography, J Chromatogr A, 1028 (2004) 219-226.

[3] J.A. Tripp, F. Svec, J.M.J. Frechet, S.L. Zeng, J.C. Mikkelsen, J.G. Santiago, Highpressure electroosmotic pumps based on porous polymer monoliths, Sensor Actuat B-Chem, 99 (2004) 66-73.

[4] F.Q. Nie, M. Macka, L. Barron, D. Connolly, N. Kent, B. Paull, Robust monolithic silica-based on-chip electro-osmotic micro-pump, Analyst, 132 (2007) 417-424.

[5] W. Wang, C.Y. Gu, K.B. Lynch, J.J. Lu, Z.Y. Zhang, Q.S. Pu, S.R. Liu, HighPressure Open-Channel On-Chip Electroosmotic Pump for Nanoflow High Performance Liquid Chromatography, Anal. Chem., 86 (2014) 1958-1964.

[6] C. He, Z. Zhu, C. Gu, J. Lu, S. Liu, Stacking open-capillary electroosmotic pumps in series to boost the pumping pressure to drive high-performance liquid chromatographic separations, J Chromatogr A, 1227 (2012) 253-258.

[7] C. Gu, Z. Jia, Z. Zhu, C. He, W. Wang, A. Morgan, J.J. Lu, S. Liu, Miniaturized Electroosmotic Pump Capable of Generating Pressures of More than 1200 Bar, Anal. Chem., 84 (2012) 9609-9614. 
[8] R.P.W. Scott, Microbore Columns in Liquid-Chromatography, J. Chromatogr. Sci., 18 (1980) 49-54.

[9] A. Chen, K.B. Lynch, X.C. Wang, J.J. Lu, C.Y. Gu, S.R. Liu, Incorporating highpressure electroosmotic pump and a nano-flow gradient generator into a miniaturized liquid chromatographic system for peptide analysis, Anal. Chim. Acta, 844 (2014) 90-98.

[10] L. Zhou, J.J. Lu, C. Gu, S. Liu, Binary electroosmotic-pump nanoflow gradient generator for miniaturized high-performance liquid chromatography, Anal Chem, 86 (2014) 12214-12219.

[11] K. Deguchi, S. Ito, S. Yoshioka, I. Ogata, A. Takeda, Nanoflow gradient generator for capillary high-performance liquid chromatography, Anal. Chem., 76 (2004) $1524-1528$.

[12] A. Premstaller, H. Oberacher, C.G. Huber, High-performance liquid chromatography-electrospray ionization mass spectrometry of single- and doublestranded nucleic acids using monolithic capillary columns, Anal. Chem., 72 (2000) 4386-4393.

[13] J. Yi, J. Heinecke, H. Tan, P.C. Ford, G.B. Richter-Addo, The distal pocket histidine residue in horse heart myoglobin directs the O-binding mode of nitrite to the heme iron, J Am Chem Soc, 131 (2009) 18119-18128.

[14] J.S. Valastyan, S. Lindquist, Mechanisms of protein-folding diseases at a glance, Disease Models \& Mechanisms, 7 (2014) 9-14.

[15] J.D. Tipton, J.C. Tran, A.D. Catherman, D.R. Ahlf, K.R. Durbin, N.L. Kelleher, Analysis of intact protein isoforms by mass spectrometry, J Biol Chem, 286 (2011) 25451-25458.

[16] S. Shiva, Nitrite: A Physiological Store of Nitric Oxide and Modulator of Mitochondrial Function, Redox Biol, 1 (2013) 40-44.

[17] U.B. Hendgen-Cotta, U. Flogel, M. Kelm, T. Rassaf, Unmasking the Janus face of myoglobin in health and disease, J Exp Biol, 213 (2010) 2734-2740.

[18] J.G. Guillemette, Y. Matsushimahibiya, T. Atkinson, M. Smith, Expression in Escherichia-Coli of a Synthetic Gene Coding for Horse Heart Myoglobin, Protein Eng., 4 (1991) 585-592.

[19] J. Zaia, B.S. Annan, K. Biemann, The Correct Molecular-Weight of Myoglobin, a Common Calibrant for Mass-Spectrometry, Rapid Commun. Mass Spectrom., 6 (1992) 32-36.

[20] M. Asensio-Ramos, J. Hernandez-Borges, A. Rocco, S. Fanali, Food analysis: A continuous challenge for miniaturized separation techniques, J. Sep. Sci., 32 (2009) 3764-3800.

[21] J.P. Kutter, Liquid phase chromatography on microchips, J Chromatogr A, 1221 (2012) $72-82$. 


\section{Figure legends}

Fig. 1. Working principle for gradient generation. (A) Schematic configuration of gradient generator using a dead-volume (mixing chamber) within a fluid conduit. (B) Detailed construction of the mixing chamber. The selection valve in (A) is used to connect the capillary assembly to a specific solution at a given time for a preset duration, while the $\mathrm{D}$ indicates an ultra-violet absorbance detector. The PEEK tubing has an i.d. of $390 \mu \mathrm{m}$, while the fused silica capillaries on both sides of the PEEK tubing have an i.d. of $75 \mu \mathrm{m}$. The length of the PEEK tubing forming the mixing chamber varies from 0 to $10 \mathrm{~mm}$; the " $0 \mathrm{~mm}$ " indicates the two pieces of fused silica capillaries are joined together. (C) An example gradient profile generated by (A). The PEEK tubing had a length of 10 $\mathrm{mm}$ (a dead-volume of $\sim 1.2 \mu \mathrm{L}$ ). E1, E2, E3, and E4 were respectively $75 \% \mathrm{ACN}+2.5 \%$ acetone, $56 \% \mathrm{ACN}+1.9 \%$ acetone, $38 \% \mathrm{ACN}+1.2 \%$ acetone, and $19 \% \mathrm{ACN}+0.6 \%$ acetone in $0.1 \%$ TFA aqueous solution. The normalized concentrations were the percentage of the highest signal recorded by D at $265 \mathrm{~nm}$ as E1, E2, E3, E4, and water were aspirated into the capillary assembly, and all the solutions were pushed back (at $~ 14$ $\min )$. The numbers on the curve indicate the aspiration duration for the specific solutions. The pump aspiration rate was $\sim 300 \mathrm{~nL} \mathrm{~min}^{-1}$, and the forward-pumping rate was $\sim 400 \mathrm{~nL}$ $\min ^{-1}$.

Fig. 2. Schematic configuration of micro HPLC. (A) Schematic configuration of micro HPLC. There were two EOPs on the left. HV was a high voltage power supply. Since the EOP monoliths were negatively charged, the electroosmotic flow (EOF) went with the 
electric field. V1 was a 12-port switching valve; one of the ports (designated by the solid circle) was blocked. $\mathrm{M}$ was a mixing chamber (see Fig. 1A and 1B). V2 was a selection valve. V3 was a 60-nL injection valve, $\mathrm{C}$ was packed capillary column, D was a UV absorbance detector (214 $\mathrm{nm}$ for peptide and protein separations or $265 \mathrm{~nm}$ for gradient profile measurements). W was designated waste reservoir. The capillaries connecting the EOP to V1 were $100 \mu \mathrm{m}$ i.d. $\times 360 \mu \mathrm{m}$ o.d. $\times 15 \mathrm{~cm}$ length, the capillary connecting V1 and V2 was $75 \mu \mathrm{m}$ i.d. $\times 360 \mu \mathrm{m}$ o.d. $\times 12 \mathrm{~cm}$ in length), and the capillary connecting V1 and V3 was $50 \mu \mathrm{m}$ i.d. $\times 360 \mu \mathrm{m}$ o.d. $\times 12 \mathrm{~cm}$ in length. MA stands for mobile phase A (5\% ACN in 0.1\% TFA). Gradient Loop and MA-Loop were fused silica capillaries having identical dimensions (100-cm-long × 250- $\mu \mathrm{m}$-i.d. $\times$ 365- $\mu \mathrm{m}$-o.d.). E1, E2, E3, E4, and $\mathrm{E} 5$ were respectively $45 \%, 27.5 \%, 15 \%, 10 \%$, and $5 \% \mathrm{ACN}$ in $0.1 \%$ TFA. (B)

Gradient profile formed using the above apparatus after removing V3 and C (see Results and Discussions for details).

Fig. 3. Chromatograms for repetitive runs. Sample- trypsin digests of BSA $\left(10 \mathrm{mg} \mathrm{mL}^{-}\right.$ $\left.{ }^{1}\right)$. When a $\mathrm{HV}$ of $-3.6 \mathrm{kV}$ was applied to the bottom EOP to aspirate five eluent solutions, the aspiration rate was $\sim 500 \mathrm{~nL} \mathrm{~min}{ }^{-1}$. When $+3.2 \mathrm{kV}$ was applied to top EOP for elution or column equilibration, the pump rate was $\sim 150 \mathrm{~nL} \mathrm{~min}^{-1}$. The $60-\mathrm{nL}$ injection valve (V3) could be set at either a "Load" position or an "Injection" position. Injection of $20 \mathrm{~nL}$ sample into $\mathrm{C}$ was implemented by switching V3 from "Load" to "Injection" and allowing it stay at "Injection" position for $8 \mathrm{~s}$, and then switching it back to "Load" position. The absorbance signal was monitored at $214 \mathrm{~nm}$. 
Fig. 4. Performance comparison between micro HPLC and Agilent 1200 system. (A) and (C), chromatograms of trypsin digests of BSA (10 $\left.\mathrm{mg} \mathrm{mL}^{-1}\right)$ and hh Mb H64V (10 mg $\mathrm{mL}^{-1}$ ) obtained from our micro HPLC. (B) and (D), chromatograms of trypsin digests of BSA (10 mg mL $\left.{ }^{-1}\right)$ and hh Mb H64V (10 mg mL $\left.{ }^{-1}\right)$ obtained from Agilent 1200 system. The same Linear UVIS 200 absorbance detector as used in Fig.3 was utilized here. The dashed lines in (A) and (C) represent the gradient profiles. The dashed lines in (B) and (D) represent the programmed gradient profiles, while the solid lines represent the gradient profiles measured between V1 and V3 using a $\mathrm{C}^{4} \mathrm{D}$ detector (see SI for details).

Fig. 5. Coupling micro HPLC with ESI-MS for real-world sample analysis. Sample mutated myoglobin (H64V) and wild type myoglobin $\left(20 \mathrm{ng} \mathrm{mL} \mathrm{m}^{-1}\right.$ each) mixture. Separation column - home-made PS-DVB monolithic column 100- $\mu$ m-i.d. $\times 365-\mu$ m-o.d. $\times 25$-cm-long. Injection volume $-10 \mathrm{~nL}$. (Note: we used a $10 \mathrm{~nL}$ injection valve here.) A $\mathrm{HV}$ of $-3.6 \mathrm{kV}$ was applied to the bottom EOP to aspirate five eluent solutions $(60 \%$, $52.5 \%, 45 \%, 37.5 \%$, and $30 \% \mathrm{ACN}$ in $0.05 \%$ TFA respectively for $10,6,5.5,5.0$, and $5.5 \mathrm{~min}$. A HV of $+4.8 \mathrm{kV}$ was applied to top $\mathrm{EOP}\left(\right.$ pump rate $=\sim 470 \mathrm{~nL} \mathrm{m^{-1 }}$ ) for elution. ESI voltage was set at $3.0 \mathrm{kV}$, and the MS was set as a full scanning mode with $\mathrm{m} / \mathrm{z}$ range of $700-2000$. Insets $\underline{\mathrm{A}}, \underline{\mathrm{B}}$, and $\underline{\mathrm{C}}$ are mass spectra of peaks $\underline{\mathrm{a}}, \underline{\mathrm{b}}$, and $\underline{\mathrm{c}}$, respectively. 
Graphic Abstract

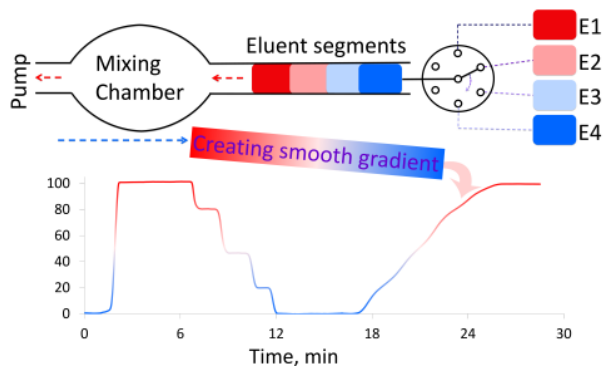



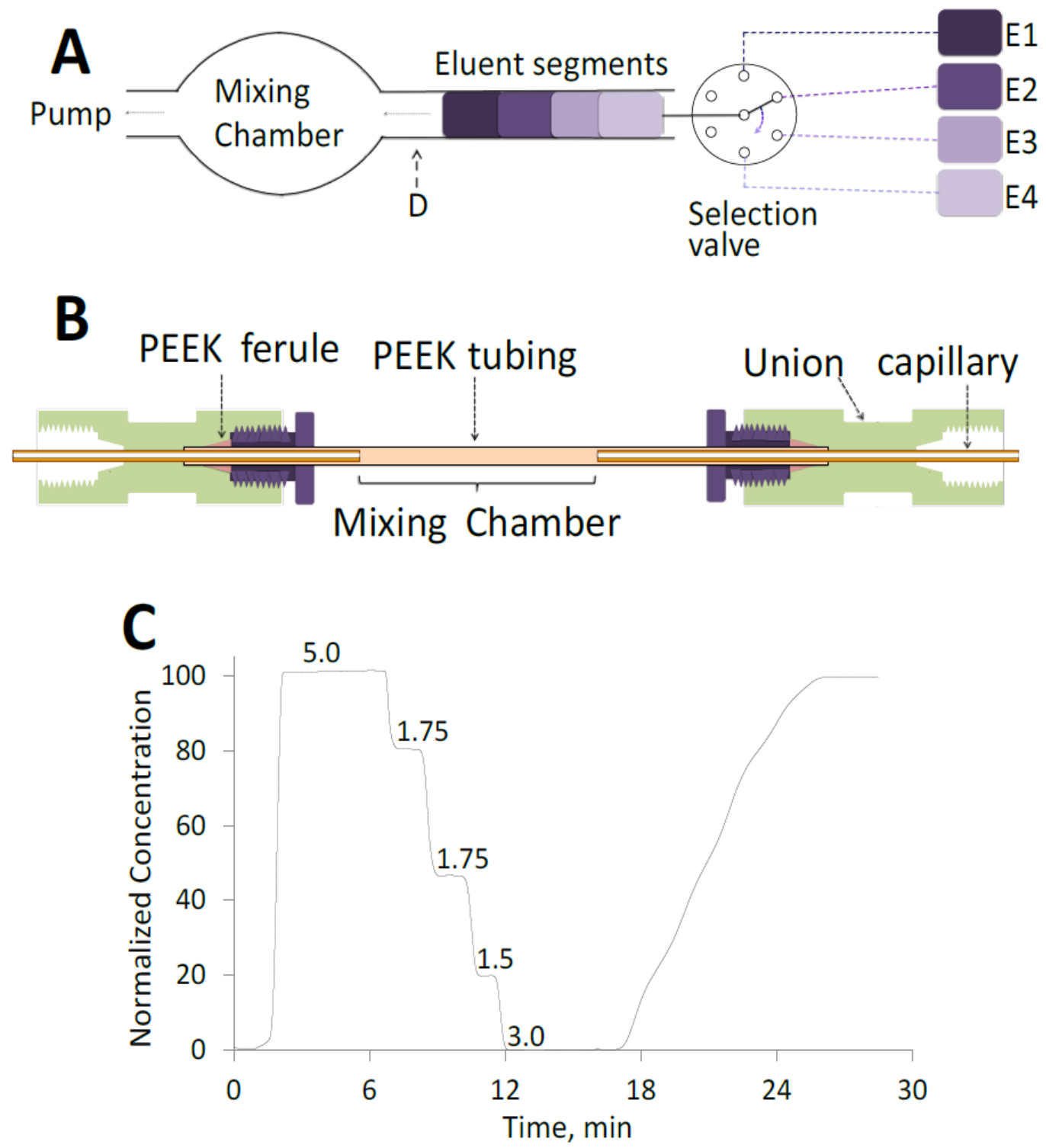

Fig. 1. 
A

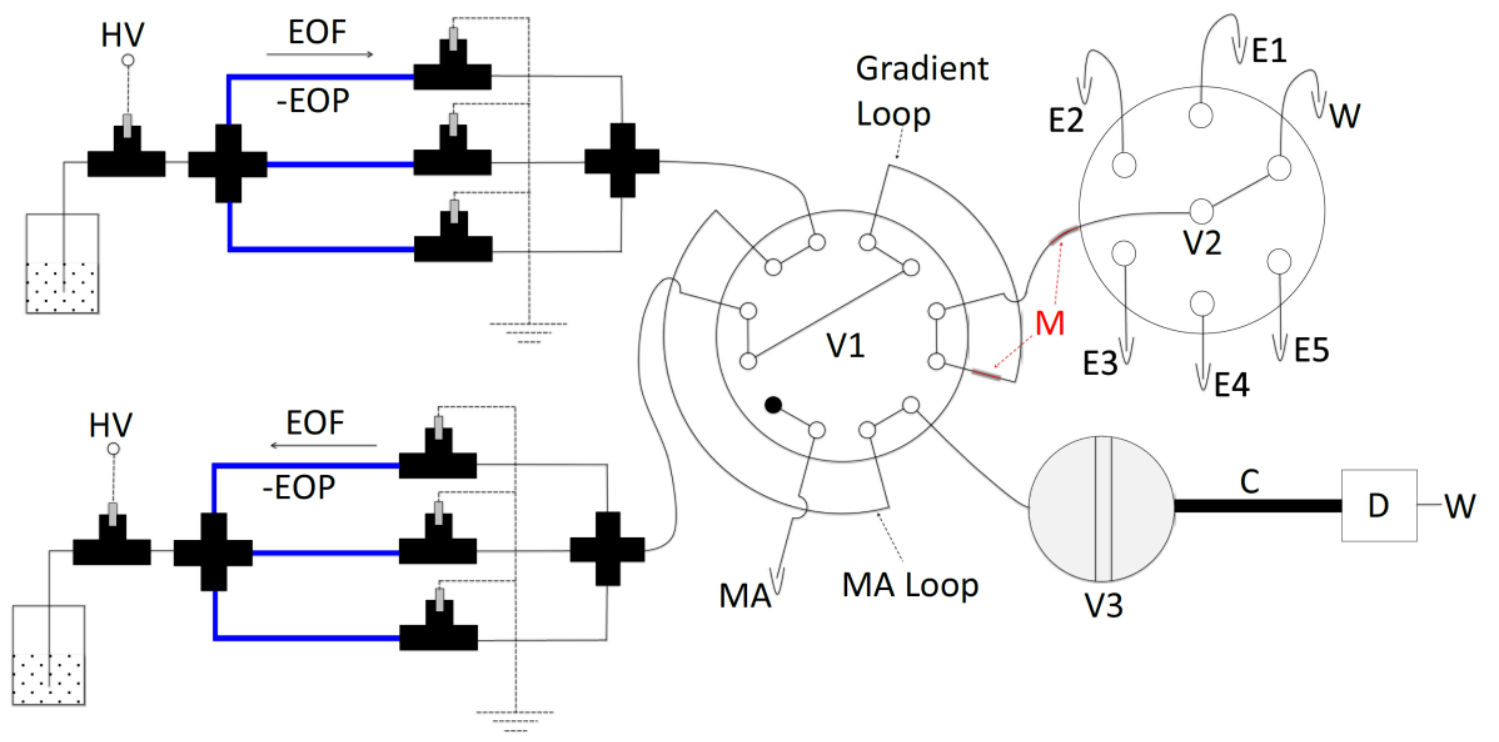

B

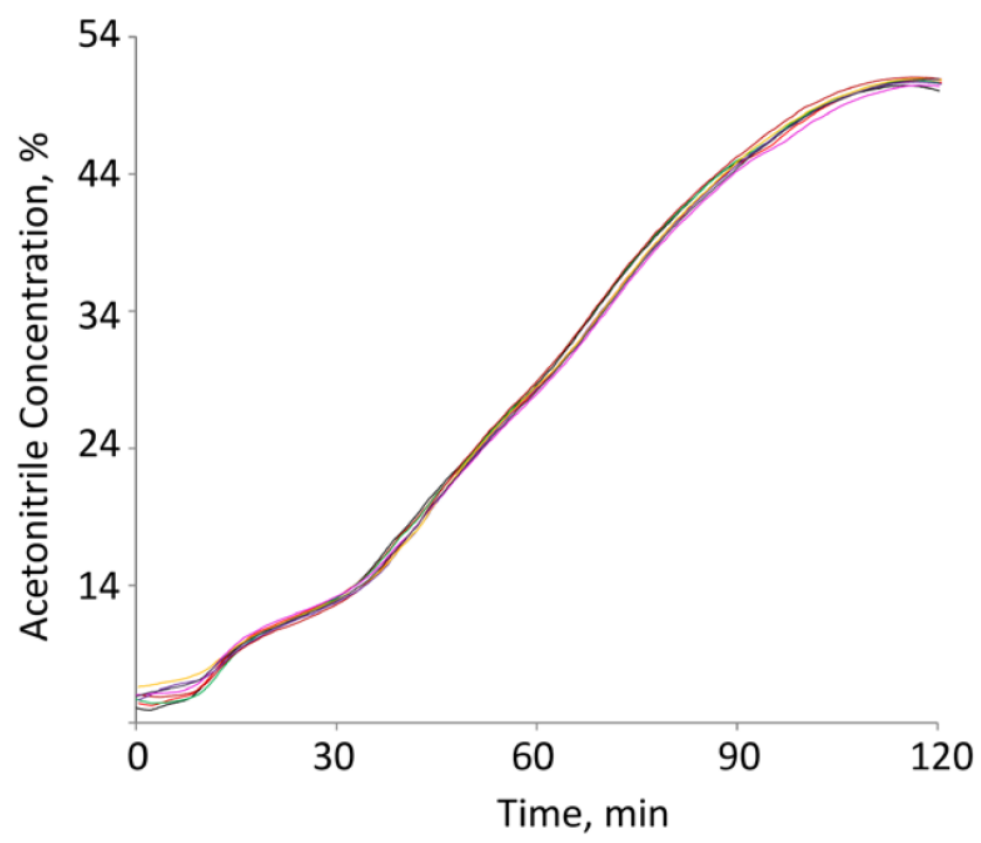

Fig. 2. 
1

2

3

4

5

6

8

9

10

11

12

13

14

15

16

17

18

19

20

21

22

23

24

25

26

27

28

29

30

31

32

33

34

35

36

37

38

39

40

41

42

43

44

45

46

47

48

49

50

51

52

53

54

55

56

57

58

59

60

61

62

63

64

65
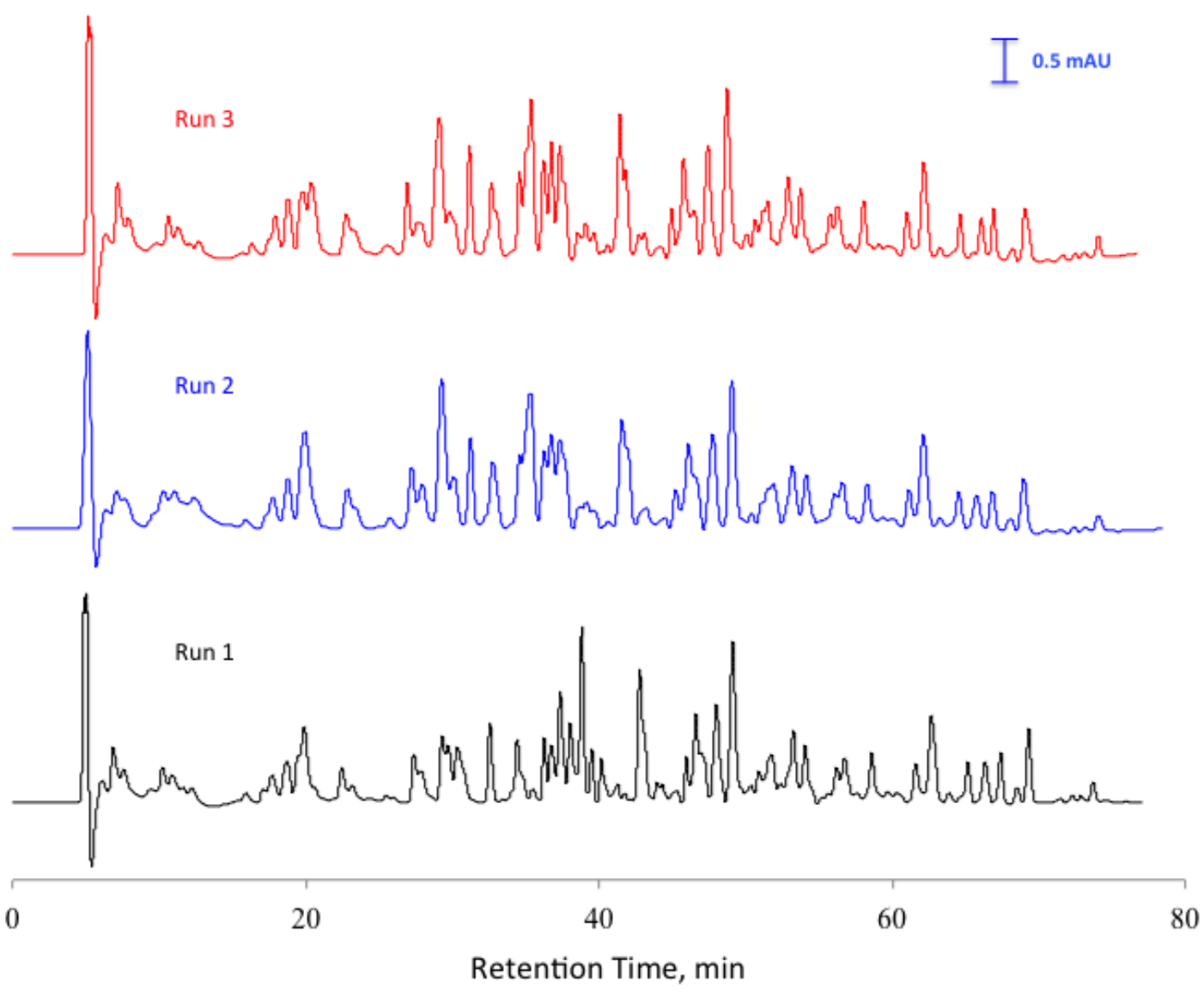

Fig. 3. 


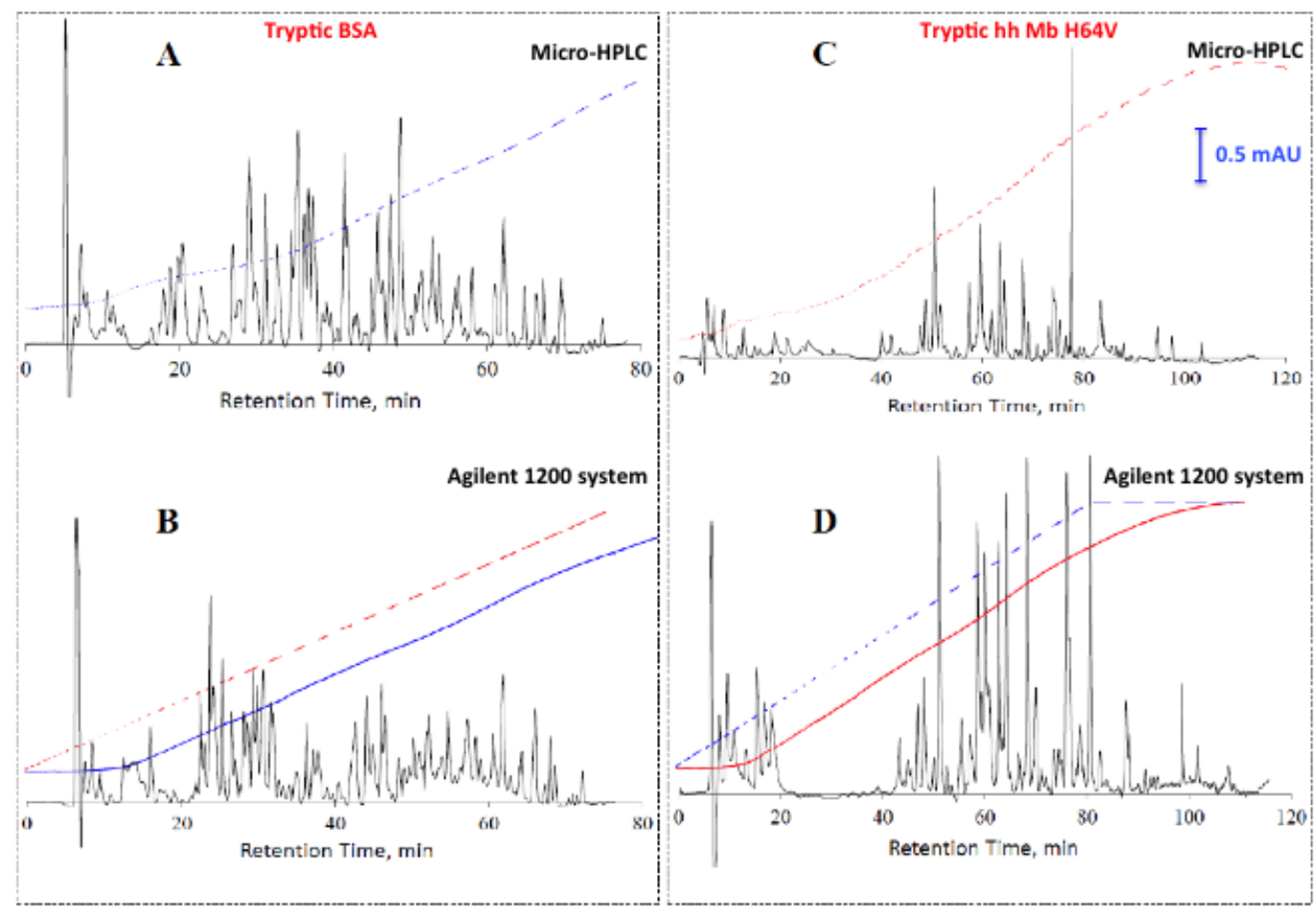

Fig. 4. 
1

2

3

4

5

6

8

9

10

11

12

13

14

15

16

17

18

19

20

21

22

23

24

25

26

27

28

29

30

31

32

33

34

35

36

37

38

39

40

41

42

43

44

45

46

47

48

49

50

51

52

53

54

55

56

57

58

59

60

61

62

63

64

65

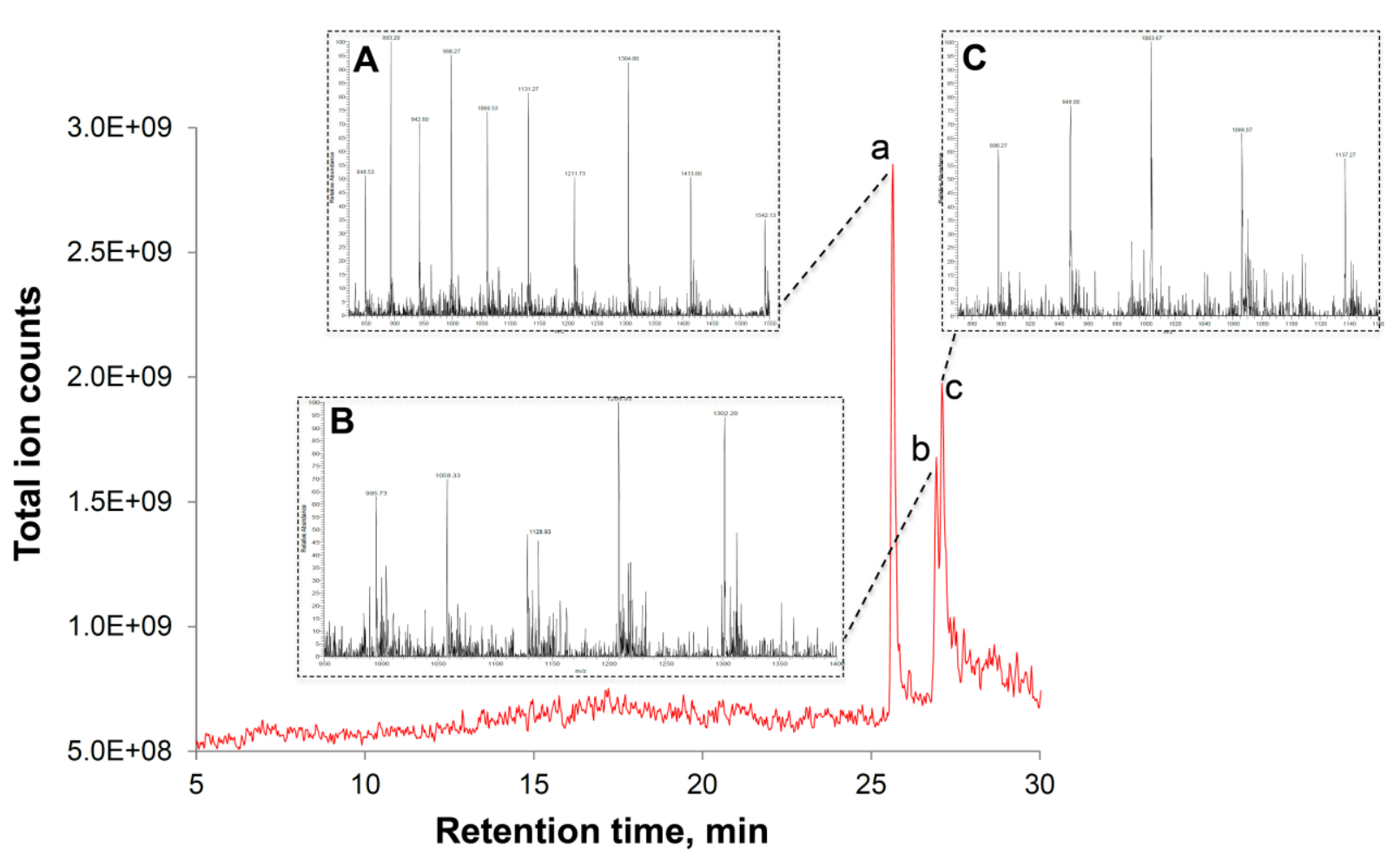

Fig. 5. 